\title{
Beyond-Luttinger-liquid thermodynamics of a one-dimensional Bose gas with repulsive contact interactions
}

\author{
Giulia De Rosi $\odot,{ }^{1,2, *}$ Pietro Massignan $\odot,{ }^{1,2, \dagger}$ Maciej Lewenstein $\odot,{ }^{1,3}$ and Grigori E. Astrakharchik $\odot^{2, \sharp}$ \\ ${ }^{1}$ ICFO - Institut de Ciencies Fotoniques, The Barcelona Institute of Science and Technology, \\ Avenida Carl Friedrich Gauss 3, 08860 Castelldefels (Barcelona), Spain \\ ${ }^{2}$ Departament de Física, Universitat Politècnica de Catalunya, Campus Nord B4-B5, 08034 Barcelona, Spain \\ ${ }^{3}$ ICREA - Institució Catalana de Recerca i Estudis Avançats, Passeig Lluís Companys 23, 08010 Barcelona, Spain
}

(Received 22 May 2019; published 7 November 2019)

\begin{abstract}
We present a thorough study of the thermodynamics of a one-dimensional repulsive Bose gas, focusing in particular on corrections beyond the Luttinger-liquid description. We compute the chemical potential, pressure, and contact as a function of temperature and gas parameter with an exact thermal Bethe ansatz. In addition, we provide interpretations of the main features in the analytically tractable regimes, based on a variety of approaches (Bogoliubov, hard core, Sommerfeld, and virial). The beyond-Luttinger-liquid thermodynamic effects are found to be nonmonotonic as a function of gas parameter. Such behavior is explained in terms of nonlinear dispersion and "negative excluded volume" effects, for weak and strong repulsion, respectively, responsible for the opposite sign corrections in the thermal next-to-leading term of the thermodynamic quantities at low temperatures. Our predictions can be applied to other systems including super Tonks-Girardeau gases, dipolar and Rydberg atoms, helium, quantum liquid droplets in bosonic mixtures, and impurities in a quantum bath.
\end{abstract}

DOI: 10.1103/PhysRevResearch.1.033083

\section{INTRODUCTION}

Gapless systems in one spatial dimension often feature a linear phononic spectrum at low momenta and this strongly constrains the low-temperature thermodynamics. A unified description of the various quantum degeneracy regimes is then obtained within Luttinger-liquid (LL) theory, which relates the low-temperature properties of the system to the Luttinger parameter, i.e., the ratio of the Fermi velocity and the zerotemperature sound velocity, itself a function of the interaction strength [1-4]. In the weakly repulsive or Gross-Pitaevskii (GP) regime, a gas of bosons with short-range interactions admits a mean-field description [5]. In the opposite limit of very strong repulsion, the gas approaches the Tonks-Girardeau (TG) limit, where bosons become impenetrable and the system wave function can be mapped onto that of an ideal Fermi gas (IFG), resulting in indistinguishable thermodynamics [6]. Seminal experiments have explored this continuous interaction crossover in the past few years [7-13].

The landscape of physical regimes in a one-dimensional (1D) Bose gas is even richer at higher temperature [14-16]. The correlation functions behave differently in the various regimes [17-20], but those are hard to access experimentally.

\footnotetext{
*giulia.derosi@icfo.eu

†pietro.massignan@upc.edu

‡grigori.astrakharchik@upc.edu
}

Published by the American Physical Society under the terms of the Creative Commons Attribution 4.0 International license. Further distribution of this work must maintain attribution to the author(s) and the published article's title, journal citation, and DOI.
Thermodynamic quantities can be measured more easily, but these generally exhibit a monotonic behavior to lowest order in temperature. For example, the phononic excitations are responsible for the linear increase with temperature of the specific heat [5] and for the quadratic growth of the chemical potential [21,22], for every interaction strength. As the temperature is increased, however, higher momenta get explored and the deviation of the spectrum from the simple linear behavior becomes important [23,24], resulting in a continuous structure bounded by two branches of elementary excitations [25]. In the GP regime, the upper particlelike branch corresponds to the Bogoliubov spectrum [26], while the lower holelike one is instead associated with the dark soliton dispersion predicted by Gross-Pitaevskii theory [27]. In the opposite TG regime, the upper and the lower branches coincide with the particle and hole excitations of the ideal Fermi gas, respectively [5]. Such complex structure has not permitted, so far, an easy physical interpretation of its effects on the corresponding thermodynamic behavior. This important gap is filled by the present work. As we will demonstrate, the resulting thermal corrections are no longer monotonic and permit one to classify the regimes of interaction.

In this paper, we provide a detailed study of the beyondLuttinger-liquid thermodynamics [24,28] in a 1D Bose gas with short-range (contact) repulsion. First, we solve numerically the thermal Bethe ansatz (TBA) equations within the Yang-Yang theory, which provide an exact answer to the problem at all temperatures $T$ and interaction strengths [29,30], and we compute key thermodynamic quantities, such as the chemical potential $\mu$, pressure $P$, and Tan's contact $\mathcal{C}$. Then, we gain further insight into the problem by investigating analytically different tractable regions, including low and high temperatures, and weak and strong interactions. We 
demonstrate that the Bogoliubov (BG) theory correctly describes thermodynamic properties at low temperatures and weak interactions. For strong repulsion, we show that the leading interaction effects at both low and high temperatures stem from a "negative excluded volume" correction derived from the hard-core (HC) model. Moreover, we demonstrate that the contact is proportional to the chemical potential in the GP limit at low temperatures and to the pressure in the TG regime for any $T$. Finally, we show that the leading beyondLL correction vs temperature in the investigated quantities (i.e., $\mu, P$, and $\mathcal{C}$ ) is negative in the GP regime and is positive in the TG limit. The same trend is also visible in the first correction to the leading classical gas contribution at high $T$.

\section{MODEL}

The Hamiltonian of a 1D gas of $N$ bosons with contact repulsive interactions is given by

$$
H=-\frac{\hbar^{2}}{2 m} \sum_{i=1}^{N} \frac{\partial^{2}}{\partial x_{i}^{2}}+g \sum_{i>j}^{N} \delta\left(x_{i}-x_{j}\right),
$$

where $m$ is the atom mass, $g=-2 \hbar^{2} /(m a)$ is the coupling constant, and $a<0$ is the 1D $s$-wave scattering length. The interaction strength is determined by the dimensionless quantity $\gamma=-2 /(n a)$ which depends on the gas parameter $n a$, with $n=N / L$ the linear density and $L$ the length of the system. There is a crossover between the weak $(\gamma \ll 1)$ and strong $(\gamma \gg 1)$ interaction limits. A peculiar feature of one dimension is that the high-density $n|a| \gg 1$ regime is described by the Bogoliubov theory contrarily to the usual three-dimensional case. Instead, the low-density $n|a| \ll 1$ limit corresponds to a unitary Bose gas where the system (1) possesses the same thermodynamic properties of an IFG.

At zero temperature, the system reduces to the LiebLiniger model, whose ground-state energy $E_{0}$, chemical potential $\mu_{0}=\left(\partial E_{0} / \partial N\right)_{a, L}$, and sound velocity $v=$ $\sqrt{n / m\left(\partial \mu_{0} / \partial n\right)_{a}}$ can be found from Bethe ansatz as a function of the interaction strength $\gamma[5,25,31]$. The speed of sound smoothly changes from the mean-field value $v_{\mathrm{GP}}=$ $\sqrt{g n / m}$ to the Fermi velocity $v_{F}=\hbar \pi n / m$ in the TG regime.

Within the canonical ensemble, the complete thermodynamics of the system is obtained starting from the Helmholtz free energy $A=E-T S$, with $E$ the energy and $S$ the entropy. This allows for the calculation of the chemical potential

$$
\mu=(\partial A / \partial N)_{T, a, L},
$$

pressure

$$
P=-(\partial A / \partial L)_{T, a, N}=n \mu-A / L,
$$

and Tan's contact parameter [32,33]

$$
\mathcal{C}=\left(4 m / \hbar^{2}\right)(\partial A / \partial a)_{T, L, N} .
$$

Simple scaling considerations [34,35] lead to a series of exact thermodynamic relations holding for any value of temperature and interaction strength (see Appendix A),

$$
-\frac{\mathcal{C}}{N} \frac{\hbar^{2} a}{4 m}=3 \frac{A}{N}+2 \frac{T S}{N}-\mu=2 \frac{E}{N}-\frac{P}{n} .
$$

The chemical potential, pressure, and contact across the whole spectrum of temperature and interaction strength, as given by the solution of the thermal Bethe ansatz equations, are shown as symbols in Figs. 1-3. The results are reported as ratios of the observables to their values given by the LL theory. With this choice, at low $T$, they all converge to unity for any value of the interaction strength $\gamma$, while, at higher $T$, any deviation from Luttinger-liquid line quantifies beyond-LL behavior which, instead, is strongly affected by $\gamma$. In these figures, we rescale the temperature by the chemical potential at zero temperature $m v^{2}$, defining $\bar{T} \equiv k_{B} T /\left(m v^{2}\right)$. Since $m v^{2}$ is also the typical energy associated with phonons, depending on $\gamma$ through the sound velocity $v$, such temperature unit is the proper one for the LL description holding in the whole interaction crossover. In the rest of the paper, we provide the understanding of dominant effects in the regimes which may be treated analytically.

\section{CHEMICAL POTENTIAL}

Let us start by considering weak interactions $(\gamma \ll 1)$. At low temperatures $k_{B} T \ll m v^{2}$, the gas behaves like a quasicondensate, exhibiting features of superfluids [36] with phononic excitations [22]. In this regime, the thermodynamics can be understood via Bogoliubov theory in terms of a gas of noninteracting bosonic quasiparticles [5]. The thermal free energy is

$$
\Delta A_{\mathrm{BG}}=A-E_{0}=k_{B} T L \int_{-\infty}^{+\infty} \frac{d p}{2 \pi \hbar} \ln \left[1-e^{-\beta \epsilon(p)}\right],
$$

where $\epsilon(p)=\sqrt{p^{2} v^{2}+\left[p^{2} /(2 m)\right]^{2}}$ is the $T=0$ BG spectrum [25,31], which depends on $\gamma$ through $v$. From Eq. (6), the temperature shift of the chemical potential is found to be

$$
\Delta \mu_{\mathrm{BG}}=\mu-\mu_{0}=\left(\frac{\partial v}{\partial n}\right)_{L} \int_{-\infty}^{+\infty} \frac{d p}{2 \pi \hbar} \frac{\partial \epsilon(p)}{\partial v} \frac{1}{e^{\beta \epsilon(p)}-1} .
$$

Within the LL theory, one retains only the phononic part of the BG dispersion, $\epsilon(p) \approx v|p|$, and obtains the universal result $\Delta \mu_{\mathrm{LL}}=\bar{\mu} \bar{T}^{2}$, with $\bar{\mu}=\pi m^{2} v^{2}(\partial v / \partial n)_{L} /(6 \hbar)$ [22]. Expanding the $\mathrm{BG}$ spectrum to higher momenta, $\epsilon(p) \approx$ $v|p|\left[1+p^{2} /\left(8 m^{2} v^{2}\right)\right]$, allows one to compute the first correction beyond LL, which is $O\left(\bar{T}^{4}\right)$ (see Appendix B 1):

$$
\Delta \mu_{\mathrm{BG}}=\Delta \mu_{\mathrm{LL}}\left[1-\pi^{2} \bar{T}^{2} / 4\right]+O\left(\bar{T}^{6}\right) .
$$

At $m v^{2} \ll k_{B} T \ll m v_{F}^{2}$, where $m v_{F}^{2}$ provides the degeneracy temperature, the gas is in the thermal degenerate state.

At even higher temperatures $k_{B} T \gg m v_{F}^{2}$, the gas behaves classically with negative chemical potential. In the GP regime, the dominant contribution to thermodynamics is determined by single-particle excitations. A reliable description in this case is provided by Hartree-Fock theory, which yields the chemical potential $\mu_{\mathrm{GP}}=\mu_{\mathrm{IBG}}+2 g n$ [5], with $\mu_{\mathrm{IBG}}$ the chemical potential of the ideal Bose gas (IBG). Hence, we perform the virial expansion of the equation of state in terms of a small effective fugacity, $\tilde{z}=e^{\beta\left(\mu_{\mathrm{GP}}-2 g n\right)} \ll 1$. At leading order in temperature, one obtains $\Delta \mu \approx \mu_{\mathrm{GP}}$, with

$$
\mu_{\mathrm{GP}}=k_{B} T[\ln (n \lambda)-n \lambda / \sqrt{2}]+O\left(T^{0}\right),
$$

where $\lambda=\sqrt{2 \pi \hbar^{2} /\left(m k_{B} T\right)}$ is the thermal wavelength. Equation (9) is an expansion for small gas parameter $n \lambda \ll 1$ 


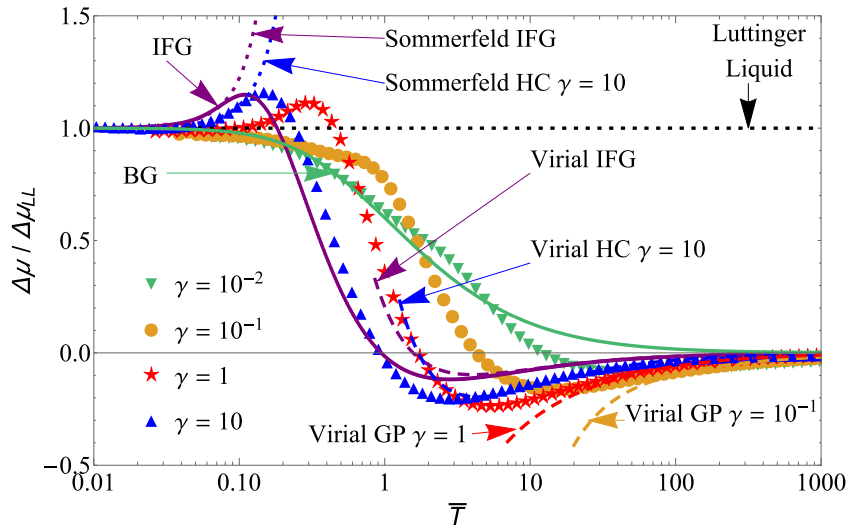

FIG. 1. Thermal shift of the chemical potential, $\Delta \mu=\mu-\mu_{0}$, vs temperature. The symbols denote numerical TBA results for several interaction strengths $\gamma$, and the lines correspond to various theories described in the text. The temperature is normalized to the typical energy associated with phonons, $\bar{T}=k_{B} T /\left(m v^{2}\right)$, and we measure shifts in terms of the LL result $\Delta \mu_{\mathrm{LL}}$, so that deviations from unity directly quantify beyond-LL effects. In these units, the $\mathrm{BG}$ result, given by Eq. (7), is independent of $\gamma$.

and it holds if $\lambda$ is much larger than the interaction range. Equation (9) depends on the coupling constant $g$ only through the $O\left(T^{0}\right)$ term (see Appendix B 2). For smaller $\gamma$ (i.e., larger densities), higher values of $\bar{T}$ are needed for the agreement of Eq. (9) with TBA, as may be seen in Fig. 1.
For strong interactions $(\gamma \gg 1)$, the thermodynamics at any temperature may be addressed by making an analogy with the hard-core model [37]. Its free energy is obtained from that of an ideal Fermi gas, subtracting from the system size an "excluded volume" $\mathrm{Na}$, where $a$ is the diameter of the HC,

$$
A_{\mathrm{HC}}(L)=A_{\mathrm{IFG}}(L \rightarrow \hat{L} \equiv L-N a) .
$$

The scattering length $a$ is positive for hard-core potentials, and the available phase space is diminished by $\mathrm{Na}$. For the repulsive $\delta$ potential in Eq. (1), instead, the scattering length is negative and the phase space is increased by $N|a|$ effectively inducing "negative excluded volume." Although the HC equation of state applies for $a>0$, its continuation to $a<0$ at $T=0$ differs from the Lieb-Liniger equation of state only by terms $O(n a)^{4}$, with such deviation attributed to the different phase shift dependence on the scattering momentum for $\delta$-function and hard-core potentials [38]. We find that the negative excluded volume correction turns out to be dominant for $\gamma \gg 1$ and permits one to describe the thermodynamics of $\delta$-interacting gas even at high $T$, as shown in Fig. 1. Similarly, we expect that the "positive excluded volume" correction will be important for the thermodynamics of short-range gases with $a>0$ in a strongly correlated metastable state (super Tonks-Girardeau gas $[39,40]$ ).

Following the Sommerfeld expansion of the IFG free energy [41] (see Appendix C 1) and taking into account the excluded volume correction for a HC gas through Eq. (10), we arrive at

$$
\mu_{\mathrm{HC}}=\hat{E}_{F}\left[\left(1+\frac{2}{3} a \hat{n}\right)+\frac{\pi^{2} \hat{\tau}^{2}}{12}(1+2 a \hat{n})+\frac{\pi^{4} \hat{\tau}^{4}}{36}\left(1+\frac{6}{5} a \hat{n}\right)+\frac{7 \pi^{6} \hat{\tau}^{6}}{144}\left(1+\frac{10}{9} a \hat{n}\right)+O\left(\hat{\tau}^{8}\right)\right]
$$

where $\hat{\tau}=k_{B} T / \hat{E}_{F}$, with an effective Fermi energy $\hat{E}_{F}=$ $\hbar^{2} \pi^{2} \hat{n}^{2} /(2 m)$ depending on the rescaled density $\hat{n}=n /(1-$ an) which takes into account the negative excluded volume and is applicable for $n|a| \ll 1$. An alternative derivation of Eq. (11) up to $O\left(T^{4}\right)$ order was already presented in Ref. [42]. We further note that our result of the IFG Sommerfeld expansion in Appendix C 1 corrects a minor misprint in the $O\left(T^{4}\right)$ term of Ref. [21]. From the $T=0$ contribution of Eq. (11), we calculate the $\mathrm{HC}$ sound velocity $v_{\mathrm{HC}}=v_{F} /(1-a n)^{2}$. By comparing Eqs. (8) and (11), one notices that the $O\left(T^{2}\right)$-phononic contribution is always positive, while quantum statistical effects are responsible for an opposite sign in $\mathrm{BG}$ and $\mathrm{HC}$ theories in the beyond-LL $O\left(T^{4}\right)$ term.

At high $T$, we apply the virial expansion to the equation of state of an IFG, and we get the corresponding expansion of the free energy (see Appendix C 2). Using Eqs. (10) and (2), we derive the virial expansion of the chemical potential of a hard-core gas,

$$
\mu_{\mathrm{HC}}=k_{B} T\left[\ln (\hat{n} \lambda)+a \hat{n}+\left(1+\frac{a \hat{n}}{2}\right) \frac{\hat{n} \lambda}{\sqrt{2}}\right]+O\left(T^{0}\right) .
$$

Equations (9) and (12) share the classical gas logarithmic term, while the second perturbative contribution $O(n \lambda)$ exhibits an opposite sign emerging from quantum statistics, whose effects become important at lower $T$. The TG regime $(\gamma=+\infty)$ is recovered from the HC model when $a=0$ and it possesses the same thermodynamic properties of an IFG.

\section{PRESSURE}

Let us now consider the thermal shift of the pressure, $\Delta P=$ $P-P_{0}$, with $P_{0}=n \mu_{0}-E_{0} / L$ the pressure at $T=0$. Within BG theory, we start from Eqs. (6) and (7) and approximate $\epsilon(p) \approx v|p|\left[1+p^{2} /\left(8 m^{2} v^{2}\right)\right]$ to obtain (see Appendix B 1 )

$$
\Delta P_{\mathrm{BG}}=\Delta P_{\mathrm{LL}}\left[1-\frac{\pi^{2} \bar{T}^{2}}{20} \frac{1+5 \chi_{\gamma}}{1+\chi_{\gamma}}\right]+O\left(\bar{T}^{6}\right)
$$

The leading-order (Luttinger-liquid) result is $\Delta P_{\mathrm{LL}}=\bar{P} \bar{T}^{2}$, where $\bar{P}=\pi m^{2} v^{3}\left(1+\chi_{\gamma}\right) /(6 \hbar)$ and $\chi_{\gamma}=[\partial v / \partial n]_{L} n / v$ depends on $\gamma$ through $v$. The LL result in Eq. (13) corrects a misprint in Ref. [43]. The virial expansion is used to obtain the pressure in the GP regime at high $T$, resulting in

$$
P_{\mathrm{GP}}=n k_{B} T[1-n \lambda /(2 \sqrt{2})]+O\left(T^{0}\right),
$$

whose detailed derivation is reported in Appendix B 2 .

Within the HC approach, the Sommerfeld expansion of the free energy, given by Eq. (10), and Eqs. (11) and (3) provide 


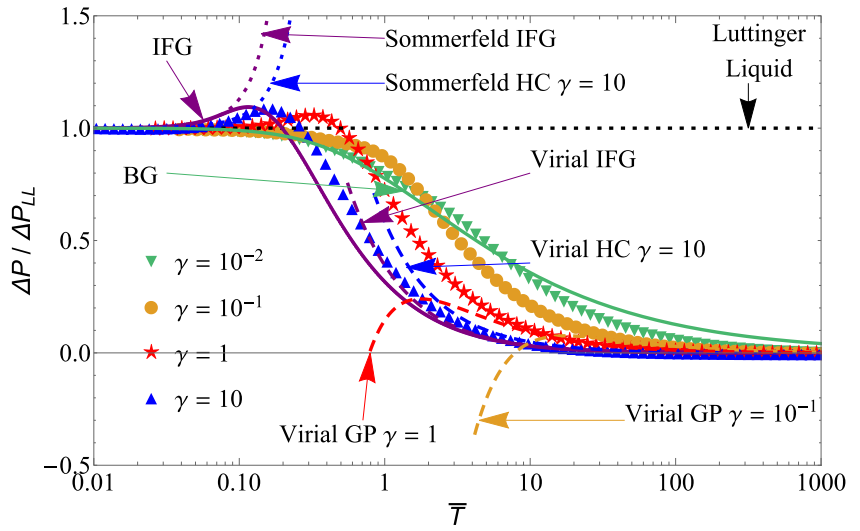

FIG. 2. Thermal shift of the pressure. Symbols denote numerical TBA results, and lines correspond to various theories, as in Fig. 1. Deviations from unity of $\Delta P / \Delta P_{\mathrm{LL}}$ indicate beyond-LL effects.

the following low- $T$ behavior of the pressure:

$$
P_{\mathrm{HC}}=\frac{2}{3} \hat{n} \hat{E}_{F}\left[1+\frac{\pi^{2}}{4} \hat{\tau}^{2}+\frac{\pi^{4}}{20} \hat{\tau}^{4}+\frac{35 \pi^{6}}{432} \hat{\tau}^{6}+O\left(\hat{\tau}^{8}\right)\right],
$$

which is consistent with Ref. [42] and includes the $O\left(T^{6}\right)$ term. The high- $T$ pressure is instead derived from the virial expansion of Eq. (10), and Eqs. (12) and (3):

$$
P_{\mathrm{HC}}=\hat{n} k_{B} T[1+\hat{n} \lambda /(2 \sqrt{2})]+O\left(T^{0}\right) .
$$

Equation (16) provides the first quantum correction to the Tonks equation $P=\hat{n} k_{B} T$, which describes a classical HC gas $[42,44,45]$, and a higher-order interaction correction to the virial result of the Yang-Yang theory [30], whose calculation is reported in Appendix D.

\section{TAN'S CONTACT}

In a system with zero-range interaction, the Tan's contact defined in Eq. (4) provides a relation between the equation of state and short-distance (large-momentum) properties, such as the interaction energy, the pair correlation function, and the relation between pressure and energy density [35,46-49], as shown, for example, in Eq. (5).

Let us compute here the thermal contribution to the contact, $\Delta \mathcal{C}=\mathcal{C}-\mathcal{C}_{0}$, where $\mathcal{C}_{0}=4 m / \hbar^{2}\left(\partial E_{0} / \partial a\right)_{L, N}$ is the contact at $T=0$. Within the BG theory, from Eq. (6) one obtains

$$
\Delta \mathcal{C}_{\mathrm{BG}}=(\overline{\mathcal{C}} / \bar{\mu}) \Delta \mu_{\mathrm{BG}},
$$

with $\overline{\mathcal{C}}=\pi m^{3} v^{2} N \gamma^{2}(\partial v / \partial \gamma)_{n} /\left(3 \hbar^{3}\right)$ entering in the LL result, $\Delta \mathcal{C}_{\mathrm{LL}}=\overline{\mathcal{C}} \bar{T}^{2}$. It can be shown that Eq. (17) is consistent with Eq. (5). At high $T$ and in the GP regime, $\mathcal{C}$ does not depend on $T$ since, within the Hartree-Fock approximation, the free energy depends on $a$ only through the $T=0$ term (see Appendix B 2).

With the HC approach, we find, from Eqs. (10) and (4),

$$
\mathcal{C}_{\mathrm{HC}}=4 m N P_{\mathrm{HC}} / \hbar^{2},
$$

where the temperature dependence is encoded in $P_{\mathrm{HC}}$, which is given in Eqs. (15) and (16) for low and high $T$, respectively. The relation between contact and pressure emerges from the

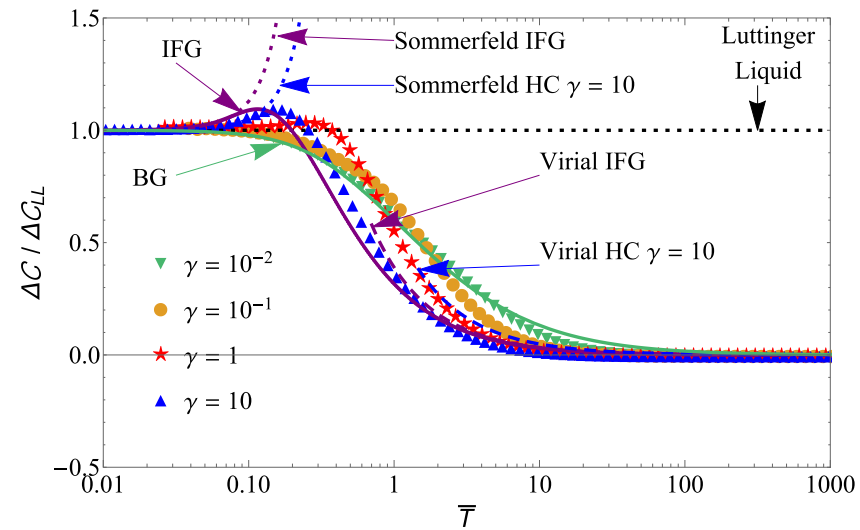

FIG. 3. Thermal shift of the Tan's contact. Symbols denote numerical TBA results and lines correspond to various theories, as in Fig. 1. Deviations from unity of $\Delta \mathcal{C} / \Delta \mathcal{C}_{\mathrm{LL}}$ indicate beyondLuttinger-liquid corrections.

HC excluded volume $\hat{L}=L-N a$, which transforms the $a$ dependence, given by Eq. (4), in a $\hat{L}$ one: $\mathcal{C}_{\mathrm{HC}} \propto P_{\mathrm{HC}}=$ $-\left(\partial A_{\mathrm{HC}} / \partial \hat{L}\right)_{T, L, N}$, holding at any $T$. Equation (18) can be derived directly from Eq. (5) by using $E_{\mathrm{HC}}=\hat{L} P_{\mathrm{HC}} / 2$.

\section{EXPERIMENTAL CONSIDERATIONS}

The pressure, chemical potential, free energy, energy, and entropy as a function of $T$ have been measured by using in situ absorption imaging in three-dimensional ultracold gases [50-52]. A similar experimental technique has been applied to a 1D Bose gas to extract the chemical potential as a function of temperature $T$ and interaction strength $\gamma$ [16], resulting in an excellent agreement with TBA. Finally, Tan's contact parameter can be extracted from radio-frequency spectroscopy [53-55], Bragg spectroscopy [56], and from the large-momentum tail of the momentum distribution $n(k)$ $[57,58]$.

\section{CONCLUSIONS}

We provided a complete study of the chemical potential, pressure, and contact as a function of temperature and interaction strength for a 1D Bose gas with repulsive contact interactions. Exact results were obtained within thermal Bethe ansatz theory and the main features were described analytically. Beyond-Luttinger-liquid effects were explained in terms of a nonlinear Bogoliubov dispersion relation for weak interactions and negative excluded volume for strong repulsion. The beyond-LL effects are responsible for an opposite sign in the thermal next-to-leading term of the low- $T$ thermodynamic behavior, being negative in the GP limit and positive in the TG regime. The same trend is also visible in the first correction to the leading classical gas contribution at high $T$. Finally, we found that the Tan's contact parameter is proportional to the chemical potential and to the pressure for weak and strong interactions, respectively.

Looking forward, our work may stimulate further theoretical and experimental investigations aimed at the characterization of quantum degeneracy regimes, the beyond-Luttingerliquid physics, and the microscopic nature of 1D Bose gases. 
Our predictions are relevant for the investigation of the properties of impurities immersed in helium [59], in a 1D Bose gas [60], and in other 1D quantum liquids [61,62] as a function of $T$ and the interaction strength of the bath. Also, the knowledge of thermodynamics is crucial for the description of harmonically trapped gases $[14,33,63,64]$, especially for the investigation of breathing modes $[40,65-70]$ whose frequency values are affected by the thermodynamic properties [71,72]. Other interesting extensions of our work include multicomponent systems [73-75] and configurations with a well-defined number of atoms [76]. The nonlinear Bogoliubov dispersion effects are expected to be seen not only for contact interactions, but also in other short-range interacting systems provided that the density is high enough to be in the mean-field regime, but not yet so large that the finite-range effects are visible. On the other hand, the "excluded volume" correction is expected to be applicable essentially to any short-range interacting system (integrable or not) at low density. For example, the excluded volume effects should be as well visible in the $a>0$ regime in (i) metastable states of gas with short-range interactions, i.e., for the super Tonks-Girardeau gas $[39,40]$, (ii) gases with finite-range interactions such as dipolar atoms [77-79], Rydberg atoms [80], bosonic ${ }^{4} \mathrm{He}$ (liquid) in a certain density range [81], and fermionic ${ }^{3} \mathrm{He}$ (gas) at low densities [82]. Our results can be extended to 1D quantum liquid droplets in bosonic mixtures [83] in order to explore thermal effects. In particular, 1D enhances quantum fluctuations [84,85], which are responsible for droplet stability, and is achieved in current experiments [86].

\section{ACKNOWLEDGMENTS}

G.D.R.'s project has received funding from the European Union's Horizon 2020 research and innovation program under the Marie Skłodowska-Curie grant agreements UltraLiquid No. 797684 and PROBIST No. 754510, and from the "A. della Riccia" Foundation. G.D.R. and M.L. acknowledge the Spanish Ministry MINECO (National Plan 15 Grants: FISICATEAMO No. FIS2016-79508-P, SEVERO OCHOA No. SEV-2015-0522, FPI), European Social Fund, Fundació Cellex, Generalitat de Catalunya (AGAUR Grant No. 2017 SGR 1341 and CERCA/Program), ERC AdG OSYRIS and NOQIA, EU FETPRO QUIC, the National Science Centre, and Poland-Symfonia Grant No. 2016/20/W/ST4/00314. P.M. is financially supported by the "Ramón y Cajal" program. G.E.A. and P.M. acknowledge funding from the Spanish MINECO (Grant No. FIS2017-84114-C2-1-P). The Barcelona Supercomputing Center (The Spanish National Supercomputing Center - Centro Nacional de Supercomputación) is acknowledged for the provided computational facilities (Grant No. RES-FI-2019-1-0006).

\section{APPENDIX A: THERMODYNAMIC RELATIONS}

In this Appendix, we provide details about the derivation of the thermodynamic relations.

Let us consider the following general expression of the free energy per particle required by dimensional analysis [34,35]:

$$
\frac{A(T, a, n)}{N} \propto n^{2} f\left(n a, \frac{T}{n^{2}}\right) \text {. }
$$

From Eq. (A1), one can deduce the scaling law

$$
\frac{A\left(\ell^{2} T, \ell^{-1} a, \ell n\right)}{N}=\ell^{2} \frac{A(T, a, n)}{N},
$$

where $\ell$ is an arbitrary, dimensionless parameter. Taking the derivative of Eq. (A2) with respect to $\ell$ at $\ell=1$ yields

$$
\begin{aligned}
& {\left[2 T\left(\frac{\partial}{\partial T}\right)_{a, L, N}-a\left(\frac{\partial}{\partial a}\right)_{T, L, N}+n\left(\frac{\partial}{\partial n}\right)_{T, a, L}\right] \frac{A(T, a, n)}{N}} \\
& =2 \frac{A(T, a, n)}{N} .
\end{aligned}
$$

From Eq. (A3) and by using Eqs. (2)-(4), and $A=E-T S$ where $S=-(\partial A / \partial T)_{a, L, N}$, we find Eq. (5).

\section{APPENDIX B: WEAKLY-INTERACTING BOSE GAS}

In this Appendix, we report the full calculation regarding the low- and high-temperature expansions of a weakly interacting Bose gas.

\section{Low-temperature expansion from nonlinear Bogoliubov dispersion relation}

The low-momentum expansion of the Bogoliubov spectrum $x=\epsilon(p)=v|p|\left[1+p^{2} /\left(8 m^{2} v^{2}\right)\right]>0$ may be inverted to find the only real and positive solution $p$. Hence, for the free energy, given by Eq. (6), we get the integral,

$$
\begin{gathered}
\int_{0}^{+\infty} d x \frac{1}{v\left[1+\frac{3 p^{2}(x)}{8(m v)^{2}}\right]} \ln \left(1-e^{-\frac{x}{k_{B} T}}\right) \\
\approx-\frac{\pi^{2}}{6} \frac{\left(k_{B} T\right)}{v}+\frac{\pi^{4}}{120} \frac{\left(k_{B} T\right)^{3}}{m^{2} v^{5}},
\end{gathered}
$$

where the analytic solution may be found expanding the integrand for $|p| \ll m|v|$, which is justified at low temperatures. We find the low- $T$ expansion of the free energy, within the Bogoliubov theory:

$$
\begin{aligned}
\Delta A_{\mathrm{BG}} & =A-E_{0} \\
& =-\frac{\pi}{6} \frac{\left(k_{B} T\right)^{2} L}{\hbar v}\left[1-\frac{\pi^{2}}{20} \frac{\left(k_{B} T\right)^{2}}{m^{2} v^{4}}+O\left(T^{4}\right)\right],
\end{aligned}
$$

where $E_{0}$ is the ground-state energy calculated within the Lieb-Liniger model at zero temperature [31].

With a similar procedure for the chemical potential, given by Eq. (7), we get the integral,

$$
\begin{gathered}
\int_{0}^{+\infty} d x \frac{p^{2}(x)}{v x\left[1+\frac{3 p^{2}(x)}{8(m v)^{2}}\right]} \frac{1}{e^{\frac{x}{k_{B} T}}-1} \\
\approx \frac{\pi^{2}}{6} \frac{\left(k_{B} T\right)^{2}}{v^{3}}-\frac{\pi^{4}}{24} \frac{\left(k_{B} T\right)^{4}}{m^{2} v^{7}},
\end{gathered}
$$

which provides the low- $T$ expansion given by Eq. (8). 


\section{High-temperature virial expansion within the Hartree-Fock theory}

The equation of state for a 1D weakly interacting Bose gas with density $n$ and pressure $P$ can be derived from

$$
\begin{aligned}
n \lambda & =g_{1 / 2}(\tilde{z}) \\
P \lambda & =g n^{2} \lambda+k_{B} T g_{3 / 2}(\tilde{z}),
\end{aligned}
$$

where $\tilde{z}=e^{\beta(\mu-2 g n)}$ is the effective fugacity within the Hartree-Fock theory [5], the Bose functions are $g_{v}(z)=\sum_{i=1}^{+\infty} z^{i} / i^{v}$, and the thermal wavelength is $\lambda=\sqrt{2 \pi \hbar^{2} /\left(m k_{B} T\right)}$.

By inverting the expression for $n$ in Eq. (B4) in terms of $\tilde{z} \ll 1$, and by expanding it for small values of the gas parameter $n \lambda \ll 1$, we obtain

$$
\tilde{z}=n \lambda-\frac{(n \lambda)^{2}}{\sqrt{2}}+\frac{\sqrt{3}-1}{\sqrt{3}}(n \lambda)^{3}+O\left[(n \lambda)^{4}\right] .
$$

By using the definition of $\tilde{z}$ in Eq. (B5) and a further expansion for $n \lambda \ll 1$, we finally find the virial expansion of the chemical potential:

$$
\begin{aligned}
\mu_{\mathrm{GP}}= & k_{B} T\left\{\ln (n \lambda)-\frac{n \lambda}{\sqrt{2}}+\frac{3 \sqrt{3}-4}{4 \sqrt{3}}(n \lambda)^{2}\right. \\
& \left.-\frac{2 \sqrt{3}-5}{6 \sqrt{2}}(n \lambda)^{3}+O\left[(n \lambda)^{4}\right]\right\}+2 g n .
\end{aligned}
$$

By considering Eq. (B5) in the equation of state of $P$, given by Eq. (B4), we derive the expansion of the pressure:

$$
\begin{aligned}
P_{\mathrm{GP}}= & n k_{B} T\left\{1-\frac{n \lambda}{2 \sqrt{2}}+\left(\frac{\gamma}{2 \pi}+\frac{3 \sqrt{3}-4}{6 \sqrt{3}}\right)(n \lambda)^{2}\right. \\
& \left.+O\left[(n \lambda)^{3}\right]\right\} .
\end{aligned}
$$

From Eqs. (B6), (B7), and (3), we calculate the high- $T$ behavior of the free energy,

$$
\begin{aligned}
A_{\mathrm{GP}}= & k_{B} T N\left\{\ln (n \lambda)-1-\frac{n \lambda}{2 \sqrt{2}}\right. \\
& \left.+\frac{3 \sqrt{3}-4}{4 \sqrt{3}} \frac{(n \lambda)^{2}}{3}+O\left[(n \lambda)^{3}\right]\right\}+g n N .
\end{aligned}
$$

We notice that at this level of approximation, the interactions appear in Eqs. (B6) and (B8) only through their contribution at zero temperature.

\section{APPENDIX C: IDEAL FERMI GAS}

In this Appendix, we provide the details of the Sommerfeld and virial expansions of an ideal Fermi gas.

The equation of state of a 1D IFG with density $n$ and pressure $P$ can be derived from

$$
\begin{aligned}
& n \lambda=f_{1 / 2}(z) \\
& P \lambda=k_{B} T f_{3 / 2}(z),
\end{aligned}
$$

where we have defined the fugacity $z=e^{\mu /\left(k_{B} T\right)}$ and the Fermi functions $f_{v}(z)=\sum_{i=1}^{+\infty}(-1)^{i-1} z^{i} / i^{v}$.

\section{Low-temperature Sommerfeld expansion}

Let us briefly review the Sommerfeld expansion [41], which enables one to calculate integrals of the form

$$
\begin{aligned}
& \int_{0}^{+\infty} d \epsilon H(\epsilon) f(\epsilon) \\
& \quad=\int_{0}^{\mu} d \epsilon H(\epsilon)+\left.\sum_{i=1}^{+\infty} a_{i}\left(k_{B} T\right)^{2 i} \frac{d^{2 i-1}}{d \epsilon^{2 i-1}} H(\epsilon)\right|_{\epsilon=\mu},
\end{aligned}
$$

where

$$
f(\epsilon)=\frac{1}{e^{\frac{\epsilon-\mu}{k_{B} T}}+1}
$$

is the Fermi-Dirac distribution. Let us, for example, consider the $1 \mathrm{D}$ density of states of the IFG,

$$
H(\epsilon)=\frac{1}{2 \sqrt{E_{F} \epsilon}},
$$

where $E_{F}=k_{B} T_{F}=\hbar^{2} \pi^{2} n^{2} /(2 m)$ is the Fermi energy. In Eq. (C2), we have introduced the dimensionless number

$$
a_{i}=\left(2-\frac{1}{2^{2(i-1)}}\right) \zeta(2 i)
$$

where $\zeta(i)$ is the Riemann zeta function.

At very low $T$, the chemical potential of the IFG approaches the Fermi energy, and hence we set $\mu \rightarrow E_{F}(1+\delta)$ with $0 \leqslant \delta \ll 1$. Then, we consider the Sommerfeld expansion, given by Eq. (C2), up to the $O\left(\delta^{3}\right)$ order, corresponding to the integer $i=3$, and we require $\int_{0}^{+\infty} d \epsilon H(\epsilon) f(\epsilon)=1$, ensuring the correct normalization. We solve the resulting equation for the real solution $\delta$ and we expand again in series, getting the chemical potential

$$
\mu_{\mathrm{IFG}}=E_{F}\left[1+\frac{\pi^{2}}{12} \tau^{2}+\frac{\pi^{4}}{36} \tau^{4}+\frac{7 \pi^{6}}{144} \tau^{6}+O\left(\tau^{8}\right)\right],
$$

with $\tau=k_{B} T / E_{F}$.

The Sommerfeld expansion, given by Eq. (C2), allows one to obtain the low-temperature behavior $k_{B} T \ll \mu$ of the Fermi functions $\quad f_{v}\left(e^{\frac{\mu}{k_{B} T}}\right) \approx \frac{1}{\nu \Gamma(v)}\left(\frac{\mu}{k_{B} T}\right)^{v}\left[1+\frac{\pi^{2}}{6} v(v-1)\left(\frac{k_{B} T}{\mu}\right)^{2}\right]$, where $\Gamma(v)$ is the Euler Gamma function. By using the latter expression in Eq. (C1), one recovers the result, given by Eq. (C6), and obtains the low-temperature expansion of the pressure,

$$
P_{\mathrm{IFG}}=\frac{2}{3} n E_{F}\left[1+\frac{\pi^{2}}{4} \tau^{2}+\frac{\pi^{4}}{20} \tau^{4}+\frac{35 \pi^{6}}{432} \tau^{6}+O\left(\tau^{8}\right)\right] .
$$

From Eqs. (C6), (C7), and (3), we calculate the low- $T$ expansion of the free energy:

$$
A_{\mathrm{IFG}}=\frac{E_{F} N}{3}\left[1-\frac{\pi^{2}}{4} \tau^{2}-\frac{\pi^{4}}{60} \tau^{4}-\frac{7 \pi^{6}}{432} \tau^{6}+O\left(\tau^{8}\right)\right] .
$$




\section{High-temperature virial expansion}

By inverting the equation for the density $n$ for $z \ll 1$, given by Eq. (C1), and by expanding for $n \lambda \ll 1$, we find

$$
z(n \lambda)=n \lambda+\frac{(n \lambda)^{2}}{\sqrt{2}}+\frac{\sqrt{3}-1}{\sqrt{3}}(n \lambda)^{3}+O\left[(n \lambda)^{4}\right],
$$

from which, using the definition of $z$ and a further expansion for $n \lambda \ll 1$, we find the virial expansion of the chemical potential,

$$
\begin{aligned}
\mu_{\mathrm{IFG}}= & k_{B} T\left\{\ln (n \lambda)+\frac{n \lambda}{\sqrt{2}}+\frac{3 \sqrt{3}-4}{4 \sqrt{3}}(n \lambda)^{2}\right. \\
& \left.+\frac{2 \sqrt{3}-5}{6 \sqrt{2}}(n \lambda)^{3}+O\left[(n \lambda)^{4}\right]\right\} .
\end{aligned}
$$

If we consider Eq. (C9) in the equation of $P$, given by Eq. (C1), we derive the high-temperature behavior of the pressure,

$$
\begin{aligned}
P_{\mathrm{IFG}}= & n k_{B} T\left\{1+\frac{n \lambda}{2 \sqrt{2}}+\frac{3 \sqrt{3}-4}{6 \sqrt{3}}(n \lambda)^{2}\right. \\
& \left.+\frac{2 \sqrt{3}-5}{8 \sqrt{2}}(n \lambda)^{3}+O\left[(n \lambda)^{4}\right]\right\},
\end{aligned}
$$

and of the free energy,

$$
\begin{aligned}
A_{\mathrm{IFG}}= & k_{B} T N\left\{\ln (n \lambda)-1+\frac{n \lambda}{2 \sqrt{2}}+\frac{3 \sqrt{3}-4}{4 \sqrt{3}} \frac{(n \lambda)^{2}}{3}\right. \\
& \left.+\frac{2 \sqrt{3}-5}{6 \sqrt{2}} \frac{(n \lambda)^{3}}{4}+O\left[(n \lambda)^{4}\right]\right\} .
\end{aligned}
$$

\section{APPENDIX D: YANG-YANG VIRIAL EXPANSION OF THE PRESSURE}

In this Appendix, we report the calculation of the YangYang virial expansion of the pressure in the weak and strong repulsion limits.

Let us consider the virial expansion of the pressure in the Yang-Yang model [30],

$$
\frac{P}{n k_{B} T}=1+\left[\frac{1}{2 \sqrt{2}}+e^{x^{2}}\left(\sqrt{\frac{2}{\pi}} \int_{0}^{x} d y e^{-y^{2}}-\frac{1}{\sqrt{2}}\right)\right](n \lambda)
$$

where $x=\gamma(n \lambda) /(2 \sqrt{2 \pi})$. Since, in Eq. (D1), only $O(n \lambda)$ terms are taken into account, we stop expansions at order $O(x)$.

In the weakly interacting regime $(x \ll 1)$, we get

$$
\frac{P_{\mathrm{GP}}}{n k_{B} T}=1-\frac{n \lambda}{2 \sqrt{2}}+\frac{\gamma}{2 \pi}(n \lambda)^{2}+O\left[(n \lambda)^{3}\right],
$$

which for $\gamma=0$ reproduces only the first correction of the virial expansion of an ideal Bose gas. We notice here that the expression we derived in Eq. (B7) is more accurate than the one in Eq. (D2), as the former contains an extra $O\left[(n \lambda)^{2}\right]$ term independent of $\gamma$, which only emerges from the nextto-leading order of the Yang-Yang virial expansion, given by Eq. (D1).

In the strong repulsive regime $(x \gg 1)$, we obtain

$$
\frac{P_{\mathrm{HC}}}{n k_{B} T}=(1+n a)+\frac{n \lambda}{2 \sqrt{2}}+O\left[(n \lambda)^{2}\right],
$$

which provides the first terms of the virial expansion of the hard-core model, given by Eq. (16).
[1] F. D. M. Haldane, Effective Harmonic-Fluid Approach to Low-Energy Properties of One-Dimensional Quantum Fluids, Phys. Rev. Lett. 47, 1840 (1981).

[2] J. Voit, One-dimensional Fermi liquids, Rep. Prog. Phys. 58, 977 (1995).

[3] T. Giamarchi, Quantum Physics in One Dimension (Oxford University Press, Oxford, 2003).

[4] M. A. Cazalilla, R. Citro, T. Giamarchi, E. Orignac, and M. Rigol, One dimensional bosons: From condensed matter systems to ultracold gases, Rev. Mod. Phys. 83, 1405 (2011).

[5] L. Pitaevskii and S. Stringari, Bose-Einstein Condensation and Superfluidity, International Series of Monographs on Physics (Oxford University Press, Oxford, 2016).

[6] M. Girardeau, Relationship between systems of impenetrable bosons and fermions in one dimension, J. Math. Phys. 1, 516 (1960).

[7] B. Paredes, A. Widera, V. Murg, O. Mandel, S. Fölling, I. Cirac, G. V. Shlyapnikov, T. W. Hänsch, and I. Bloch, TonksGirardeau gas of ultracold atoms in an optical lattice, Nature (London) 429, 277 (2004).

[8] T. Kinoshita, T. Wenger, and D. S. Weiss, Observation of a onedimensional Tonks-Girardeau gas, Science 305, 1125 (2004).

[9] B. Laburthe Tolra, K. M. O’Hara, J. H. Huckans, W. D. Phillips, S. L. Rolston, and J. V. Porto, Observation of Reduced Three-
Body Recombination in a Correlated 1D Degenerate Bose Gas, Phys. Rev. Lett. 92, 190401 (2004).

[10] T. Kinoshita, T. Wenger, and D. S. Weiss, Local Pair Correlations in One-Dimensional Bose Gases, Phys. Rev. Lett. 95, 190406 (2005).

[11] E. Haller, M. Rabie, M. J. Mark, J. G. Danzl, R. Hart, K. Lauber, G. Pupillo, and H.-C. Nägerl, Three-Body Correlation Functions and Recombination Rates for Bosons in Three Dimensions and One Dimension, Phys. Rev. Lett. 107, 230404 (2011).

[12] T. Jacqmin, J. Armijo, T. Berrada, K. V. Kheruntsyan, and I. Bouchoule, Sub-Poissonian Fluctuations in a 1D Bose Gas: From the Quantum Quasicondensate to the Strongly Interacting Regime, Phys. Rev. Lett. 106, 230405 (2011).

[13] V. Guarrera, D. Muth, R. Labouvie, A. Vogler, G. Barontini, M. Fleischhauer, and H. Ott, Spatiotemporal fermionization of strongly interacting one-dimensional bosons, Phys. Rev. A 86, 021601(R) (2012).

[14] D. S. Petrov, G. V. Shlyapnikov, and J. T. M. Walraven, Regimes of Quantum Degeneracy in Trapped 1D Gases, Phys. Rev. Lett. 85, 3745 (2000).

[15] A. Vogler, R. Labouvie, F. Stubenrauch, G. Barontini, V. Guarrera, and H. Ott, Thermodynamics of strongly correlated one-dimensional Bose gases, Phys. Rev. A 88, 031603(R) (2013). 
[16] F. Salces-Carcoba, C. J. Billington, A. Putra, Y. Yue, S. Sugawa, and I. B. Spielman, Equations of state from individual onedimensional Bose gases, New J. Phys. 20, 113032 (2018).

[17] K. V. Kheruntsyan, D. M. Gangardt, P. D. Drummond, and G. V. Shlyapnikov, Pair Correlations in a Finite-Temperature 1D Bose Gas, Phys. Rev. Lett. 91, 040403 (2003).

[18] G. E. Astrakharchik and S. Giorgini, Correlation functions and momentum distribution of one-dimensional Bose systems, Phys. Rev. A 68, 031602(R) (2003).

[19] P. Deuar, A. G. Sykes, D. M. Gangardt, M. J. Davis, P. D. Drummond, and K. V. Kheruntsyan, Nonlocal pair correlations in the one-dimensional Bose gas at finite temperature, Phys. Rev. A 79, 043619 (2009).

[20] M. Panfil and J.-S. Caux, Finite-temperature correlations in the Lieb-Liniger one-dimensional Bose gas, Phys. Rev. A 89, 033605 (2014).

[21] G. Lang, F. Hekking, and A. Minguzzi, Dynamic structure factor and drag force in a one-dimensional strongly interacting Bose gas at finite temperature, Phys. Rev. A 91, 063619 (2015).

[22] G. De Rosi, G. E. Astrakharchik, and S. Stringari, Thermodynamic behavior of a one-dimensional Bose gas at low temperature, Phys. Rev. A 96, 013613 (2017).

[23] F. Meinert, M. Panfil, M. J. Mark, K. Lauber, J.-S. Caux, and H.-C. Nägerl, Probing the Excitations of a Lieb-Liniger Gas from Weak to Strong Coupling, Phys. Rev. Lett. 115, 085301 (2015).

[24] N. Fabbri, M. Panfil, D. Clément, L. Fallani, M. Inguscio, C. Fort, and J.-S. Caux, Dynamical structure factor of onedimensional Bose gases: Experimental signatures of beyondLuttinger-liquid physics, Phys. Rev. A 91, 043617 (2015).

[25] E. H. Lieb, Exact analysis of an interacting Bose gas. II. The excitation spectrum, Phys. Rev. 130, 1616 (1963).

[26] P. P. Kulish, S. V. Manakov, and L. D. Faddeev, Comparison of the exact quantum and quasiclassical results for a nonlinear Schrödinger equation, Theor. Math. Phys. 28, 615 (1976).

[27] M. Ishikawa and H. Takayama, Solitons in a One-Dimensional Bose System with the Repulsive Delta-Function Interaction, J. Phys. Soc. Jpn. 49, 1242 (1980).

[28] A. Imambekov, T. L. Schmidt, and L. I. Glazman, Onedimensional quantum liquids: Beyond the Luttinger liquid paradigm, Rev. Mod. Phys. 84, 1253 (2012).

[29] C. N. Yang and C. P. Yang, Thermodynamics of a onedimensional system of bosons with repulsive delta-function interaction, J. Math. Phys. 10, 1115 (1969).

[30] C. P. Yang, One-dimensional system of bosons with repulsive $\delta$-function interactions at a finite temperature $T$, Phys. Rev. A 2, 154 (1970).

[31] E. H. Lieb and W. Liniger, Exact analysis of an interacting Bose gas. I. The general solution and the ground state, Phys. Rev. 130, 1605 (1963).

[32] E. Braaten, D. Kang, and L. Platter, Universal Relations for Identical Bosons from Three-Body Physics, Phys. Rev. Lett. 106, 153005 (2011).

[33] H. Yao, D. Clément, A. Minguzzi, P. Vignolo, and L. SanchezPalencia, Tan's Contact for Trapped Lieb-Liniger Bosons at Finite Temperature, Phys. Rev. Lett. 121, 220402 (2018).

[34] A. Fetter and J. Walecka, Quantum Theory of Many-Particle Systems, Dover Books on Physics Series (Dover, New York, 1971).
[35] M. Barth and W. Zwerger, Tan relations in one dimension, Ann. Phys. 326, 2544 (2011).

[36] G. E. Astrakharchik and L. P. Pitaevskii, Motion of a heavy impurity through a Bose-Einstein condensate, Phys. Rev. A 70, 013608 (2004).

[37] M. Motta, E. Vitali, M. Rossi, D. E. Galli, and G. Bertaina, Dynamical structure factor of one-dimensional hard rods, Phys. Rev. A 94, 043627 (2016).

[38] G. E. Astrakharchik, J. Boronat, I. L. Kurbakov, Y. E. Lozovik, and F. Mazzanti, Low-dimensional weakly interacting Bose gases: Nonuniversal equations of state, Phys. Rev. A 81, 013612 (2010).

[39] G. E. Astrakharchik, J. Boronat, J. Casulleras, and S. Giorgini, Beyond the Tonks-Girardeau Gas: Strongly Correlated Regime in Quasi-One-Dimensional Bose Gases, Phys. Rev. Lett. 95, 190407 (2005).

[40] E. Haller, M. Gustavsson, M. J. Mark, J. G. Danzl, R. Hart, G. Pupillo, and H.-C. Nägerl, Realization of an excited, strongly correlated quantum gas phase, Science 325, 1224 (2009).

[41] N. Ashcroft and N. Mermin, Solid State Physics, HRW Intl. Ed. (Holt, Rinehart and Winston, New York, 1976).

[42] M. Wadati, G. Kato, and T. Iida, Statistical mechanics of quantum integrable systems, in Nonlinear Waves: Classical and Quantum Aspects, edited by F. K. Abdullaev and V. V. Konotop (Springer Netherlands, Dordrecht, 2005), pp. 193-207.

[43] J. Yu-Zhu, C. Yang-Yang, and G. Xi-Wen, Understanding many-body physics in one dimension from the Lieb-Liniger model, Chin. Phys. B 24, 050311 (2015).

[44] L. Tonks, The complete equation of state of one, two and threedimensional gases of hard elastic spheres, Phys. Rev. 50, 955 (1936).

[45] D. C. Mattis, The Many-Body Problem (World Scientific, Singapore, 1993).

[46] M. Olshanii and V. Dunjko, Short-Distance Correlation Properties of the Lieb-Liniger System and Momentum Distributions of Trapped One-Dimensional Atomic Gases, Phys. Rev. Lett. 91, 090401 (2003).

[47] S. Tan, Energetics of a strongly correlated Fermi gas, Ann. Phys. 323, 2952 (2008).

[48] S. Tan, Generalized virial theorem and pressure relation for a strongly correlated Fermi gas, Ann. Phys. 323, 2987 (2008).

[49] S. Tan, Large momentum part of a strongly correlated Fermi gas, Ann. Phys. 323, 2971 (2008).

[50] T.-L. Ho and Q. Zhou, Obtaining the phase diagram and thermodynamic quantities of bulk systems from the densities of trapped gases, Nat. Phys. 6, 131 (2009).

[51] S. Nascimbène, N. Navon, K. J. Jiang, F. Chevy, and C. Salomon, Exploring the thermodynamics of a universal Fermi gas, Nature (London) 463, 1057 (2010).

[52] M. J. H. Ku, A. T. Sommer, L. W. Cheuk, and M. W. Zwierlein, Revealing the superfluid lambda transition in the universal thermodynamics of a unitary Fermi gas, Science 335, 563 (2012).

[53] R. J. Wild, P. Makotyn, J. M. Pino, E. A. Cornell, and D. S. Jin, Measurements of Tan's Contact in an Atomic Bose-Einstein Condensate, Phys. Rev. Lett. 108, 145305 (2012).

[54] Y. Sagi, T. E. Drake, R. Paudel, and D. S. Jin, Measurement of the Homogeneous Contact of a Unitary Fermi Gas, Phys. Rev. Lett. 109, 220402 (2012). 
[55] Z. Yan, P. B. Patel, B. Mukherjee, R. J. Fletcher, J. Struck, and M. W. Zwierlein, Boiling a Unitary Fermi Liquid, Phys. Rev. Lett. 122, 093401 (2019).

[56] S. Hoinka, M. Lingham, K. Fenech, H. Hu, C. J. Vale, J. E. Drut, and S. Gandolfi, Precise Determination of the Structure Factor and Contact in a Unitary Fermi Gas, Phys. Rev. Lett. 110, 055305 (2013).

[57] J. T. Stewart, J. P. Gaebler, T. E. Drake, and D. S. Jin, Verification of Universal Relations in a Strongly Interacting Fermi Gas, Phys. Rev. Lett. 104, 235301 (2010).

[58] R. Chang, Q. Bouton, H. Cayla, C. Qu, A. Aspect, C. I. Westbrook, and D. Clément, Momentum-Resolved Observation of Thermal and Quantum Depletion in a Bose Gas, Phys. Rev. Lett. 117, 235303 (2016).

[59] J. Bardeen, G. Baym, and D. Pines, Effective interaction of $\mathrm{He}^{3}$ atoms in dilute solutions of $\mathrm{He}^{3}$ in $\mathrm{He}^{4}$ at low temperatures, Phys. Rev. 156, 207 (1967).

[60] B. Reichert, A. Petković, and Z. Ristivojevic, Field-theoretical approach to the Casimir-like interaction in a one-dimensional Bose gas, Phys. Rev. B 99, 205414 (2019).

[61] A. Recati, J. N. Fuchs, C. S. Peca, and W. Zwerger, Casimir forces between defects in one-dimensional quantum liquids, Phys. Rev. A 72, 023616 (2005).

[62] M. Schecter and A. Kamenev, Phonon-Mediated Casimir Interaction between Mobile Impurities in One-Dimensional Quantum Liquids, Phys. Rev. Lett. 112, 155301 (2014).

[63] P. Vignolo and A. Minguzzi, Universal Contact for a TonksGirardeau Gas at Finite Temperature, Phys. Rev. Lett. 110, 020403 (2013).

[64] W. Xu and M. Rigol, Universal scaling of density and momentum distributions in Lieb-Liniger gases, Phys. Rev. A 92, 063623 (2015).

[65] H. Moritz, T. Stöferle, M. Köhl, and T. Esslinger, Exciting Collective Oscillations in a Trapped 1D Gas, Phys. Rev. Lett. 91, 250402 (2003).

[66] H. Hu, G. Xianlong, and X.-J. Liu, Collective modes of a one-dimensional trapped atomic Bose gas at finite temperatures, Phys. Rev. A 90, 013622 (2014).

[67] B. Fang, G. Carleo, A. Johnson, and I. Bouchoule, QuenchInduced Breathing Mode of One-Dimensional Bose Gases, Phys. Rev. Lett. 113, 035301 (2014).

[68] X.-L. Chen, Y. Li, and H. Hu, Collective modes of a harmonically trapped one-dimensional Bose gas: The effects of finite particle number and nonzero temperature, Phys. Rev. A 91, 063631 (2015).

[69] A. I. Gudyma, G. E. Astrakharchik, and M. B. Zvonarev, Reentrant behavior of the breathing-mode-oscillation frequency in a one-dimensional Bose gas, Phys. Rev. A 92, 021601(R) (2015).

[70] G. De Rosi and S. Stringari, Hydrodynamic versus collisionless dynamics of a one-dimensional harmonically trapped Bose gas, Phys. Rev. A 94, 063605 (2016).
[71] G. E. Astrakharchik, Local density approximation for a perturbative equation of state, Phys. Rev. A 72, 063620 (2005).

[72] G. De Rosi and S. Stringari, Collective oscillations of a trapped quantum gas in low dimensions, Phys. Rev. A 92, 053617 (2015).

[73] L. Yang, L. Guan, and H. Pu, Strongly interacting quantum gases in one-dimensional traps, Phys. Rev. A 91, 043634 (2015).

[74] J. Decamp, J. Jünemann, M. Albert, M. Rizzi, A. Minguzzi, and P. Vignolo, High-momentum tails as magnetic-structure probes for strongly correlated $\mathrm{SU}(\kappa)$ fermionic mixtures in one-dimensional traps, Phys. Rev. A 94, 053614 (2016).

[75] J. Decamp, P. Armagnat, B. Fang, M. Albert, A. Minguzzi, and P. Vignolo, Exact density profiles and symmetry classification for strongly interacting multi-component Fermi gases in tight waveguides, New J. Phys. 18, 055011 (2016).

[76] H. Labuhn, D. Barredo, S. Ravets, S. de Léséleuc, T. Macrì, T. Lahaye, and A. Browaeys, Tunable two-dimensional arrays of single Rydberg atoms for realizing quantum Ising models, Nature (London) 534, 667 (2016).

[77] A. S. Arkhipov, G. E. Astrakharchik, A. V. Belikov, and Y. E. Lozovik, Ground-state properties of a one-dimensional system of dipoles, J. Expt. Theor. Phys. Lett. 82, 39 (2005).

[78] R. Citro, E. Orignac, S. De Palo, and M. L. Chiofalo, Evidence of Luttinger-liquid behavior in one-dimensional dipolar quantum gases, Phys. Rev. A 75, 051602(R) (2007).

[79] M. D. Girardeau and G. E. Astrakharchik, Super-TonksGirardeau State in an Attractive One-Dimensional Dipolar Gas, Phys. Rev. Lett. 109, 235305 (2012).

[80] O. N. Osychenko, G. E. Astrakharchik, Y. Lutsyshyn, Y. E. Lozovik, and J. Boronat, Phase diagram of Rydberg atoms with repulsive van der Waals interaction, Phys. Rev. A 84, 063621 (2011).

[81] G. Bertaina, M. Motta, M. Rossi, E. Vitali, and D. E Galli, One-Dimensional Liquid ${ }^{4} \mathrm{He}$ : Dynamical Properties beyond Luttinger-liquid Theory, Phys. Rev. Lett. 116, 135302 (2016).

[82] G. E. Astrakharchik and J. Boronat, Luttinger-liquid behavior of one-dimensional ${ }^{3} \mathrm{He}$, Phys. Rev. B 90, 235439 (2014).

[83] D. S. Petrov, Quantum Mechanical Stabilization of a Collapsing Bose-Bose Mixture, Phys. Rev. Lett. 115, 155302 (2015).

[84] D. S. Petrov and G. E. Astrakharchik, Ultradilute LowDimensional Liquids, Phys. Rev. Lett. 117, 100401 (2016).

[85] P. Zin, M. Pylak, T. Wasak, M. Gajda, and Z. Idziaszek, Quantum Bose-Bose droplets at a dimensional crossover, Phys. Rev. A 98, 051603(R) (2018).

[86] P. Cheiney, C. R. Cabrera, J. Sanz, B. Naylor, L. Tanzi, and L. Tarruell, Bright Soliton to Quantum Droplet Transition in a Mixture of Bose-Einstein Condensates, Phys. Rev. Lett. 120, 135301 (2018). 